\title{
IMPLEMENTATION OF HEART RATE SENSOR AND MOTION SENSORS BASED ON INTERNET OF THINGS FOR ATHLETE PERFORMANCE MONITORING
}

\author{
${ }^{a}$ Sritrusta Sukaridhoto, ${ }^{b}$ Muhammad Aksa Hidayat Yani, ${ }^{c}$ Achmad Basuki, ${ }^{d}$ Riyadh \\ Arridha, ${ }^{\mathrm{e}}$ Andi Roy, ${ }^{\mathrm{f}}$ Titing Magfirah, ${ }^{\mathrm{g}}$ Agus Prasetyo, ${ }^{\mathrm{h}}$ Udin Harun Al Rasyid \\ ,g,h Politeknik Elektronika Negeri Surabaya, 60111 ,Surabaya, Indonesia \\ b,d,e,f Politeknik Negeri Fakfak, West Papua, Indonesia \\ E-mail: ${ }^{a}$ dhoto@pens.ac.id, ${ }^{b}$ aksahidayat@gmail.com, ${ }^{c}$ basuki@pens.ac.id, \\ driyadh.arridha@gmail.com, ${ }^{\mathrm{e}}$ andiroy177@gmail.com, ${ }^{\mathrm{f}}$ titin.magfirah@gmail.com, \\ gagus@student.pens.ac.id, hudinharun@pens.ac.id
}

\begin{abstract}
Indonesian athlete achievements in the ASEAN Games continued to decline in obtaining medals. Starting from 1962 to 2014, the medal accomplisment went down from 51 to 20 medals. The achievement decrease was due to the lack of athletic resources and the absence of media that could record athletes' skills in the field. The system can record the athletes' performance before, while and after running using the Heart Rate sensor and Motion Capture sensor that can be implemented in the field. The results of the sensor recording will be stored in the database. This system applies the Internet of Things (IoT) concept, using Raspberry Pi, Arduino Microcontroller, T34 Polar Heart Rate sensor to capture and send heartbeat to receivers, gyro-based motion-capture sensors that named wearnotch. This sensor serves to capture the movement of athletes. The sensors communicate with the system using $4 G$ connectivity and MQTT as edge computing which act as a communication medium from sensors to databases, while Maria $D B$ and influx $D B$ as accumulation play a role in storing heart rate and athlete's movements that have been recorded by sensors. Then, athlete performance monitoring platform with a heart rate sensor and athletes' motion capture is a web-based application that collaborates all processes from the sensors to the system. The results of heart rate sensor recordings are categorized good because the error margin is only $0.4 \%$.
\end{abstract}

Keywords: Motion Capture, Heartrate, IoT, monitoring, athlete. 


\section{INTRODUCTION}

Indonesia is a country with the largest population that will continue to grow every year[1]. This population increase is characterized by an increase in the population of the younger generation. The youth as the next generation must improve achievements in the field of sports, especially in the athletic branch[2]. However, the fact that Indonesia's athletes' medals achievement at the Asian Games in 1962 continued to decreasenat Asian Games in 2014 from 50 to 20 medals. In the 2018 ASEAN Games, Indonesia won 3 medals at athletic field, but Indonesia has not been able to donate a gold medal yet. This decline was caused by a lack of monitoring and evaluation of athletic resources Basically, an athlete is influenced by five things: strength, endurance, running ability, running technique, running experience, and the trainer's ability to provide learning[2][3].

Position and movement in running affect running speed. In a previous study, which measured the ratio of heart rate of amateur and professional soccer athletes, it was found that the intensity of exercise would affect changes of body abilities in the cardiovascular system. This is also emphasized by other studies[4]. There are differences in Heart Rate Variability (HRV) rhythms between athletes and nonathletes[5]. The main things in an athlete's performance are body position, athlete's movements, and heart rate rhythm.

Previous research measured heart rate using the ESP 8266 Module, Finger Grove Sensor and Arduino integrating hardware and software for monitoring heart rate [6], the shortcomings of this study were using finger grove sensors where these sensors were less effective for athletes when running in the field due to running start of the athlete performs a jumping start movement. The athlete's wrists and fingers will touch the field so that it disturbs the sensor located on the finger and also in the data storage, the data is stored in the local database where data must be uploaded manually.

The other research on the design of portable BPM was equipped with data storage. The working principle used by the tool is IR and Photodiode sensors; the location of IR and Photodiode is reflected parallel to the tip of finger. IR emits infrared light to the fingertips and the photodiode as a light receiver. Light intensity is influenced by the concentration of blood at the fingertips. Data used to calculate BPM per 3 minutes is seven times; the stored data can be recalled after the measurement has completed. The drawback of this study is that, it cannot store more data to get a better diagnosis[6]. Delivery of auscultation signals can also be carried out by using wireless sensor networks that use the Zigbee standard protocol. Zigbee networks have the advantage of flexibility in coverage and flexibility in network development because this protocol is based on a mesh network[7]. However, the shortcomings of the Zigbee network are the maximum bandwidth that can be accommodated only at $250 \mathrm{~KB}$ so that the signal delay generated is quite long. In this study, we used Polar T34 with wireless communication.

In the previous research, water quality monitoring program has been made in shrimp pond areas using the principle of IoT and Big Data where sensors are able to communicate with the Rest protocol to MQTT [8], monitoring water quality is strengthened by further research on intelligent systems monitoring water conditions and air conditions around with IoT and big data in real-time[9]. Based on the research, the research wants to apply the same concept in building its server platform, but it will be applied to monitor the performance of athletes by using different sensors and platforms.

In previous studies, the use of motion capture to help recognize language sign for the deaf, the study only applies motion capture to only capture hand motion[10], the authors propose the use of motion capture sensors at every point on the body to capture the athlete's movements.

Departing from these problems, innovation is needed to make the athlete's movement monitoring platform as well as monitor heart rate conditions that can be used directly in the field so that the trainer can see the performance of athletes who are trained and can draw conclusions - motion Catch.

In this paper, we proposed the platform that can record the performance of athletes before, while and after running using the Heart Rate sensor and Motion Capture sensor. The contribution of this research is to introduce a new methodology that integrates the 
wearnotch and heart rate sensors for athletes. The results of the sensor recordings will be stored in the database. This system applies the Internet of Things (IoT) concept[11] namely: (1) using Raspberry Pi, Arduino Microcontroller, T34 Polar Heart Rate sensor to capture and send heartbeats to receivers, sensors gyro-based motion-capture called wearnotch where these sensors function to capture the movement of athletes, (2) sensors communicate with the system using 4G connectivity, (3) the use of MQTT as edge computing which acts as a communication medium from sensors to databases, (4) Maria DB and influx DB as accumulation play a role in storing data heart rate and athlete movements that have been recorded by sensors, (5) Athletes' performance monitoring platform with heart rate sensors and athletes' motion capture are web-based applications that collaborate all processes from sensors until it is displayed to the system.

\section{MATERIAL AND METHODS}

In this paper, we proposed a platform that can measure an athlete's motion ability and cardio ability by using motion capture and heart rate sensors.

\section{System Design}

In this study, the focus is on the case of track which the movement and heart rate of the athlete will be captured directly in the field by the motion and heart rate sensors. Next, it will be sent to the Big Data Server and the data recorded, then is visualized in a web form. The system design is built as in Figure 1.

The Athletes Performance Monitoring Platform design system, this system consists of three-part, the first part is the motion capture sensor and the heart rate sensor, the second part is the data storage section using big data server, and the third part is visualization in the form of a website.

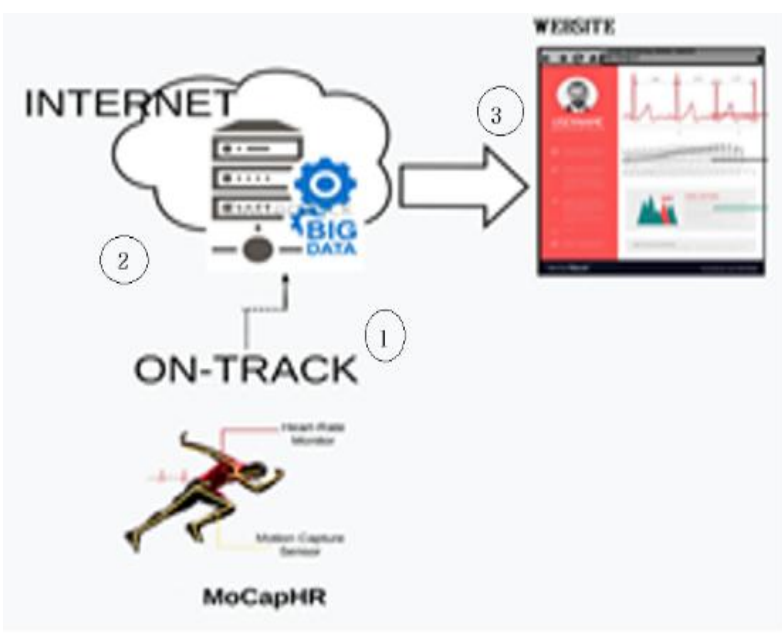

Fig 1. System Design SMAP

\section{Server Design}

The Server for Athlete Performance Monitoring Platform is built using three computer servers with multinode clustering schemes. Multinode clustering is a schema to maximize server capabilities that are integrated to deal with the problem of limited storage space, CPU, and memory[8][12].

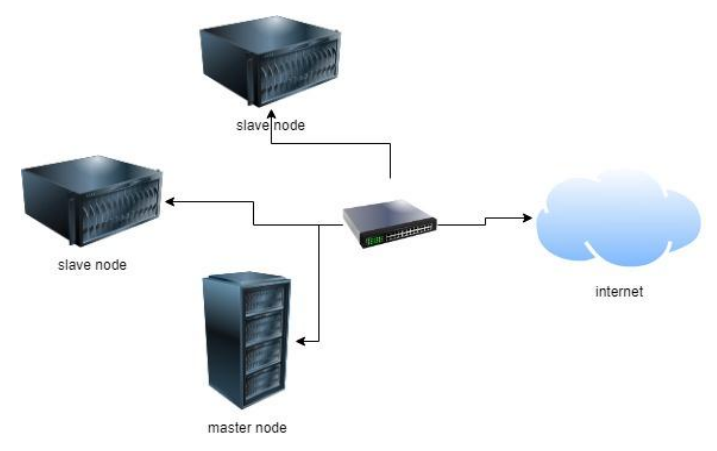

Fig 2. Server Design SMAP

In figure 2, the master node acts as a central point, which regulates the process of distributing data, allocating CPU and memory for the jobs that will be executed. The 3 servers consist of $2 \mathrm{CT}$ and $1 \mathrm{VM}$. LXC (Linux Containers) is an operating systemlevel virtualization method to run multiple Linux isolated systems (containers) on host controls using a single Linux kernel. Applying CT to PROXMOX is almost the same as creating a VM[13][14], but there are some differences. The container is a lightweight software package that can stand alone, which includes everything needed to run it: code, runtime, system tools, system libraries, 
settings. CT software will always run the same, regardless of the environment[14]. With the implementation of multi-node clusters, the problem will be limited in storage space, CPU, and memory so that it can be resolved. Later, if the four servers are still lack, it can be increased simply by adding nodes on the slave side so that it will not interfere the system as a whole.

The motion sensor used is Wearnotch to retrieve Data from the Wearnotch SDK through their server. It is one of the obstacles to getting data from the motion sensor. To overcome this problem, drawing cloud data uploaded from the cloud provided by the Wearnotch sensor, a fetcher program is required to collect the data needed. The fetcher function is to retrieve data from the sensor provider. The authors use the SDK and cloud services from Wearnotch to facilitate data retrieval. Then for data retrieval, the application is used as a fetcher/scraper to get data from the Wearnotch cloud service. In this application, the authors plan to use the Python programming language because it is proven to be reliable for memos.

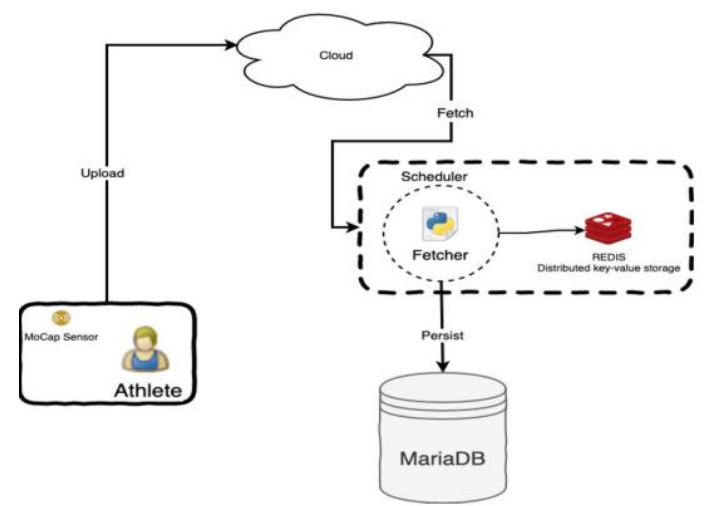

Fig 3. The fetcher application architecture

In figure 3, Data sent is text-based with data between sensors separated by commas in sequence: Sensor ID, Heartrate, Date, Time. In the MQTT protocol, the sensor node acts as MQTT Publisher, which is the party that sends data to the MQTT broker (server)[8][15]. MQTT communication uses port 1883.

MQTT broker here consists of 2 components, namely mosquito as MQTT broker and a python program as MQTT exporter which will forward data from MQTT broker to InfluxDB database. If it is seen in the picture above, there will be several MQTT broker servers and a TCP load balancer in front of it. It aims to divide data from many sensors into existing cluster MQTT, for example; 3. Mosquitto itself as MQTT broker does not support the clustering feature. Therefore, the authors develop a tool using the python programming language as the exporter of each server MQTT[15]. Then, there is a time-series database InfluxDB in the behind, this database is used to store data sent through sensors

\section{Heart Rate Sensor design}

One part of the athletes monitoring platform is heart rate monitoring, the heart rate monitoring system is built using polar heart rate sensors, raspberry pi, and Arduino. The polar heart rate sensor functions to catch the heartbeat, flat shape, and can send data through the Bluetooth.

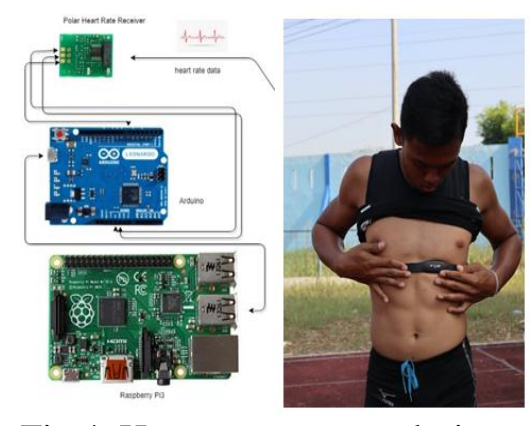

Fig 4. Heart rate sensors design

In figure 4 , we can see that the heart rate sensor is mounted on the chest, the heart rate data will be received by the polar recipient and forwarded to the Arduino, then it is forwarded to Raspberry Pi to be stored and sent to the database via the internet or wireless data. Next, the steps of the heart rate sensor will be explained in table 1.

Table 1. The algorithm on the Heartrate sensor

Algorithm 1 Heart Rate Procedure

1. Begin

2. SensorInfo <- sensorID, Heartrate

3. Loop:

4. $\quad$ Time <- System time

5. SensorData <- ECG

6. Send sensorInfo, time, sensor

Data to MQTT Server

$7 . \quad$ Save local

8. Sleep (5)

9. Goto loop

10. End 
From Table 1, there are several processes, namely the sensor info retrieval process in the form of sensor ID, Time, and Heartrate from the location of the sensor node. After that, the looping process is carried out to take system time and sensor data using pySerial. All data is then sent using the MQTT protocol and then stored locally as data backup.

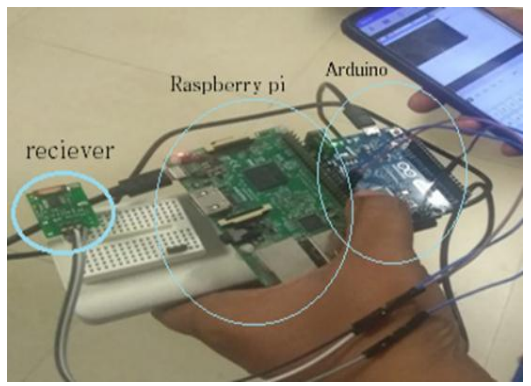

Fig 5. Heartrate sensor monitoring

Figure 5 is a series of tools to monitor heart rate, and the tool is composed of polar heart rate transmitter sensors, sensor receivers, Arduino, and Raspberry Pi. The receiver will receive Heartrate data from the transmitter that is placed on the athlete's body, as shown in figure 5 . The heart rate data received by the receiver is then forwarded to Arduino and sent serially. When the heart rate data is received by the receiver which is then forwarded to Arduino, Arduino will check the USB connection and serial data. Then, it will be converted to the string value and transferred to the raspberry pi.

\section{Design of Motion Capture Sensors}

Motion capture sensors are sensors that are used to capture the motion of an object[16]. In this study, we used wearnotch with gyro sensor and Bluetooth communication to capture athletes' motion by attaching it to the body parts which is determined by the motion sensor used, it was Wearnotch as seen in figure 6 . The principle of working the wearnotch is similar to a beacon with bluetooth wireless communication that is able to streamline energy[17].

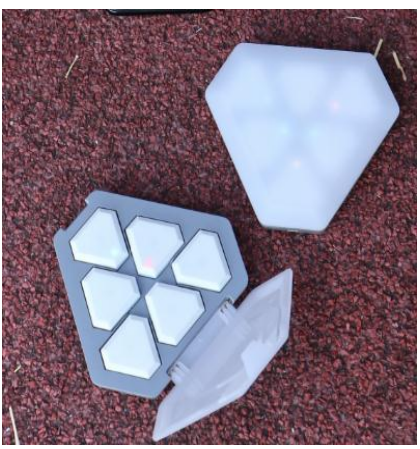

Fig 6.Wearnotch sensor

Figure 7 shows the system flow of Wearnotch. By default, Wearnotch will save the recorded data to Wearnotch Cloud so that to display it into the system that has been created; we need several other applications.

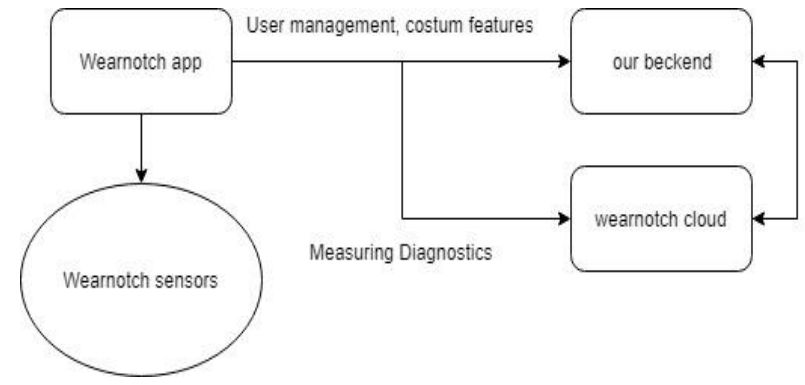

Fig 7. Wearnotch System flow

The application that will be built has several functions, including fetcher, net exporter, and a web interface that are used for administration of data in the database.

According to figure 3, it can be seen that every time the fetcher is run, it will open a connection to the MariaDB database as the primary storage[18]. Meanwhile, Redis as a system locking to make sure no other fetchers are running, if the connection fails, fetcher will try every few times. After initializing the connection to MariaDB and Redis, the fetcher will take the DO_FETCH key from Redis, if the value returned is 0 , then the fetcher will end the task, but if the value is 1 , fetcher will continue the task. The first task that this fetcher must do is to make a connection to Wearnotch and authenticate in order to be able to enter the sensor admin page. For the authentication process using a token generated from Google Auth, this token is stored in the environment variable container where the fetcher is run or can also be stored in Redis. 

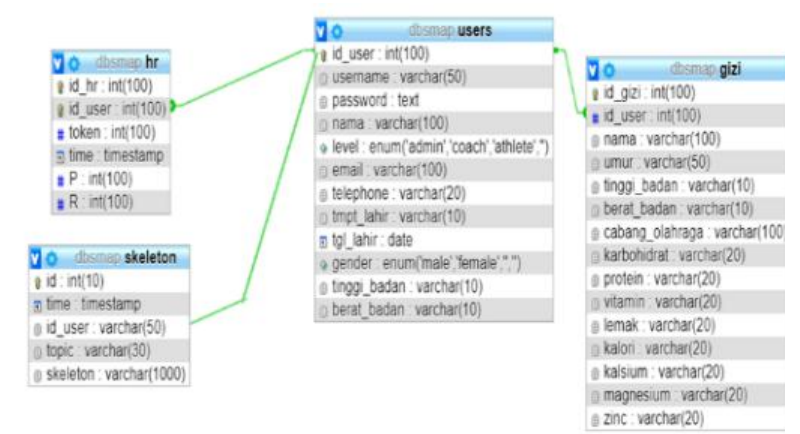

Fig 8. Design Database

When you have successfully logged in and entered the wearnotch page, the fetcher will retrieve the page data in the form of HTML text. After that, the parsing process is carried out to get the data needed, among others: time to retrieve sensor data, URL to get raw data sensors and URLs for 3D visualisation. After the required data is successfully parsed, it will be saved to an object in python and do a check to the database whether the data already exists or not, if it turns out the data already exists, then the data will not be saved, but if not, the data is collected to MariaDB. The first process is, run every time the exporter runs is that it will open a connection to MQTT as a brokered message that is connected to the sensor and influx DB as the main storage place, if the connection fails, the exporter will try every few times. After connecting to MQTT is available, the exporter will subscribe to a special topic and set a listener every time the topic has a new message. The task of the listener is to parse the MQTT message sent by the sensor and then send it to InfluxDB after the tag is given according to the athlete's name and id that was active at that time. For names and id athletes who are practising are arranged through a web interface that will be developed using Laravel. The name and id will be stored in the bush Redis with the key-value format so that it can be accessed and modified easily by the application created.

\section{Website Platform Design}

The first step to build a system is by designing Use Case; this use case diagram will describe an interaction between one or more actors with the system to be created, this diagram is used to find out what functions are in a system and who is entitled use these functions[19].
In this system, there are 3 actors, namely Admin, Coach, and Athlete.

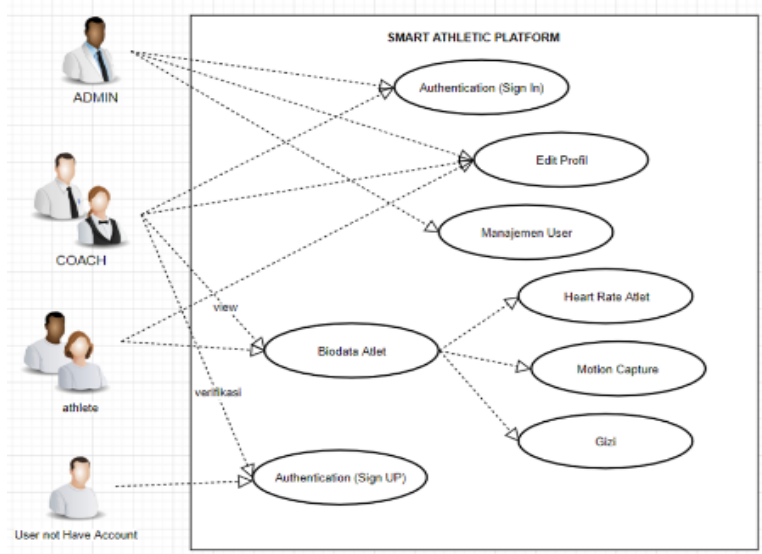

Fig 9. Usecase Diagram

In figure 9, this system consists of Admin, Coach, and Athlete. Each of these users has a role, namely:

a. Admin : Authentication (Sign In), Edit Profile, User Management

b. Athlete : Authentication (Sign In), Profile Management (Biodata) Heart Rate, Motion Capture, Nutrition, Sign Up

c. Admin : Authentication (Sign In), Heart Rate Profile Management, Motion Capture, Nutrition, Athlete Verification

\section{RESULT AND DISCUSSION}

\section{Heart Rate Sensor Performance Measurement}

At this stage, researchers have conducted research on what if Heartrate Sensor monitoring is used at different distances. This trial was conducted to determine in what extent this Heartrate sensor can send data.

Table 2. Trials the distance of heart rate sensors

\begin{tabular}{ccc} 
No & Range & Status \\
\hline 1 & $10-15$ meters & Read \\
\hline 2 & $16-25$ meters & Read \\
\hline 3 & $26-30$ meters & Read \\
\hline 4 & $31-40$ meters & Read \\
\hline 5 & $41-55$ meters & Read \\
\hline 6 & $55-60$ meters & Read \\
\hline 7 & $61-70$ meters & Read,Lost \\
\hline 8 & $71+$ & lost
\end{tabular}


From table 2, it can be concluded that the receiver sensor heart rate cannot be placed at the beginning of the 100-meter track, it should be placed in the middle of the 100-meter running track or the sensor receiver is attached to the track with a scrab.

\section{Compare the Performance of the Heart Rate Sensor with Other Censored Censors}

Trial comparison of the T34 polar sensor, Xiaomi Mi Band 3, and the Pulse Finger Sensor, this test is carried out for a comparison between the T34 sensor and the pulse finger sensor in reading heart rate data.

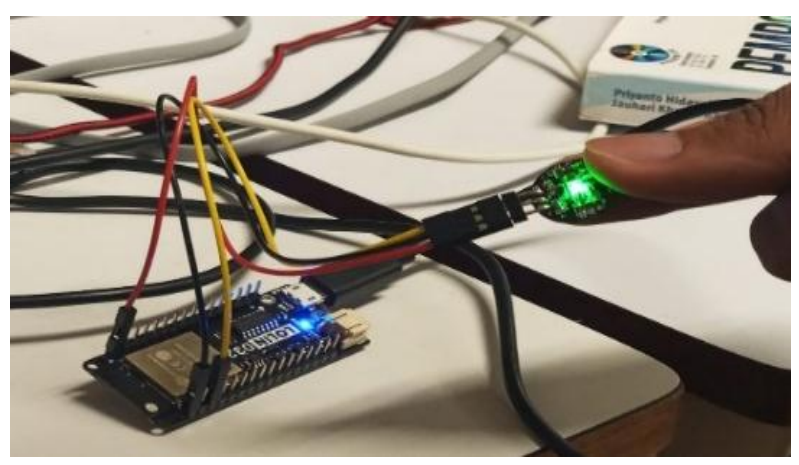

Fig 10. Pulse finger grove sensor.

Figure 10 shows Pulse Finger Sensor, is a heart rate sensor that works using the principle of Photoplethysmography (PPG) [20], which is a method to measure heart rate based on the volume of blood flow in the veins that are very close to the skin. Mi Band 3 as seen in figure 11 , is a smartwatch that can capture heart rate, has the same working principle as pulse finger sensors.

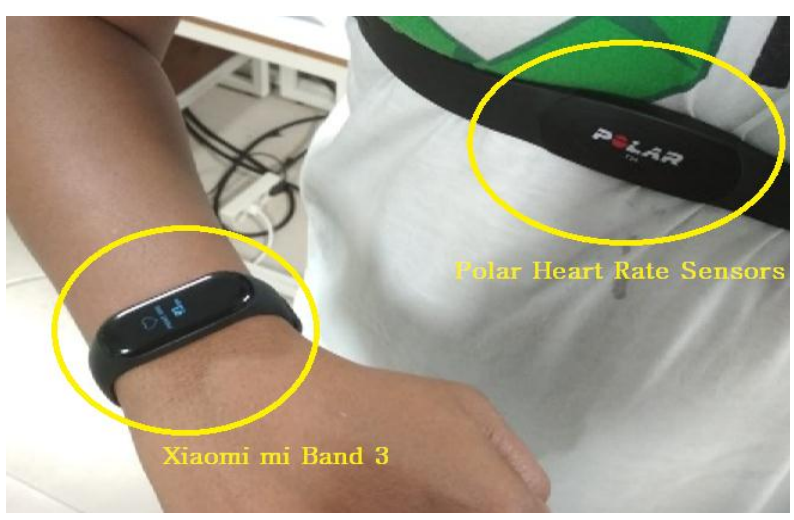

Fig 11. Heart rate sensors
The three sensors are measured on the same object to check the level of accuracy and comparison of recording results.

Table 3. The trial of heart rate sensor

\begin{tabular}{ccccccc}
$\mathrm{N}$ & Sampl & Age & $\begin{array}{c}\text { Pol } \\
\text { ar } \\
\text { (bp } \\
\text { m) }\end{array}$ & $\begin{array}{c}\text { Fing } \\
\text { er } \\
(\mathrm{bpm}\end{array}$ & $\begin{array}{c}\text { Xiao } \\
\text { mi } \\
(\mathrm{bpm}\end{array}$ & $\begin{array}{c}\text { Error } \\
(\%)\end{array}$ \\
\hline 1 & $\mathrm{~A}(\mathrm{~L})$ & 25 & 98 & 97 & 98 & 1 \\
\hline 2 & $\mathrm{~B}(\mathrm{~L})$ & 27 & 92 & 92 & 92 & 0 \\
\hline 3 & $\mathrm{C}(\mathrm{L})$ & 27 & 91 & 90 & 90 & 2 \\
\hline 4 & $\mathrm{D}(\mathrm{L})$ & 32 & 91 & 91 & 90 & 1 \\
\hline 5 & $\mathrm{E}(\mathrm{L})$ & 21 & 72 & 72 & 72 & 0 \\
\hline \multicolumn{7}{c}{ Average } \\
\end{tabular}

Table 3 shows the comparison of recording result. Based on the polar heart rate sensor test with the finger pulse sensors and xiaomi sensor, the measurement results are relatively same with an error rate of $0.4 \%$. In other words, the polar heart rate sensor is recommended for taking heart rate data because of durable vibration and can be used in the field very well.

\section{Motion capture sensors}

Each athlete and participant will be paired with eleven wearnotch sensors and one sensor heartrate.

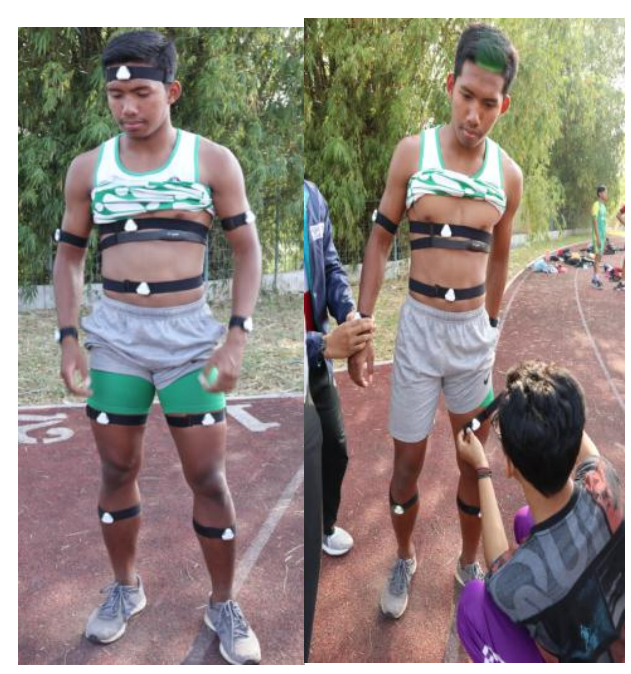

Fig 12. Sensor location in the athlete body. 
In figure 12, the sensor is placed on 11 parts of the head, chest, right upper arm, left forearm, left upper arm, left forearm, waist, right thigh, left thigh, right leg, left leg.

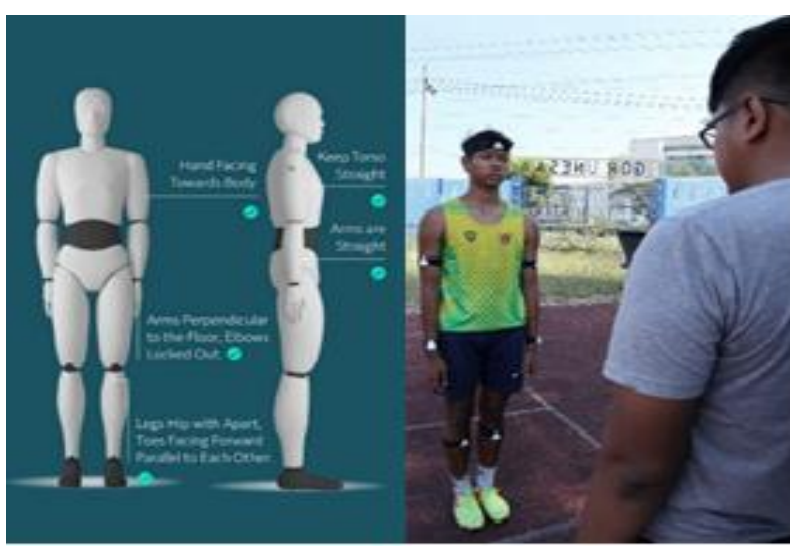

Fig 13. Sensor location in the athlete body

In figure 13 , is a sensor calibration process with the device. When a position shift occurs during calibration, the movement recorded by the sensor will not match the athlete's movements when practicing. If the calibration fails, it will affect the results of data retrieval from the motion sensor[10][21]. When a position shift occurs during calibration, the movements recorded by the sensor do not match the athlete's movements, as shown in figure 14.

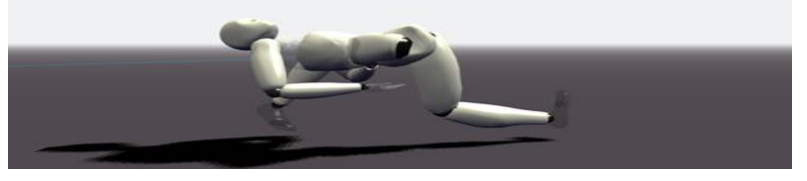

Fig 14. False recorded.

In figure 15. is an example of a successful calibration. When performing the calibration, there is no shift in the position of the sensor. Thus, the 3D animation display is by the actual testing situation. Then, the user will run 100 meters with a 3 point start position or run. After the user runs, the sensor will send data to the web for evaluation.

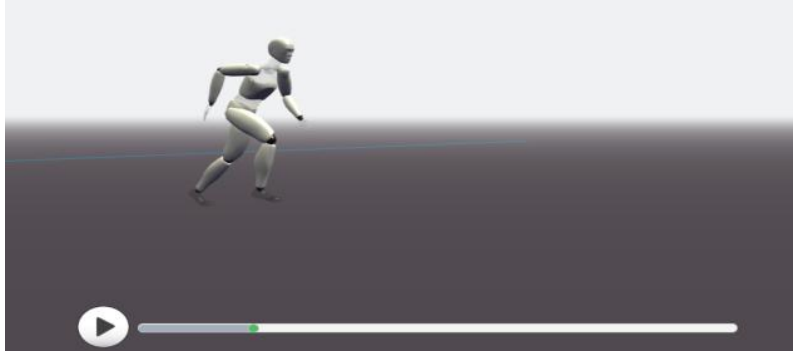

Fig 15. Successful calibration results

\section{Parameter experiment}

In this study, we conducted factual data collection in the Field of Surabaya State University campus (UNESA) at the Faculty of Sport Sciences (FIK) consisting of 10 participants. The 100-meter athletic run came from the Indonesian National Sports Committee (KONI) located at UNESA Jl. Tongue Wetan. 10 KONI entertains these athletes and do intensive training every day according to the guidance and direction of the trainer, as well as ten non-athletes were taken from Surabaya State Electronic Polytechnic Campus (PENS) consisting of 4 Students of the Computer Engineering Department, 4 Postgraduate Students and 2 Students Multimedia Broadcasting (MMB).

Data retrieval is done by using two types, namely sensor heart rate and motion capture sensor. The procedure for collecting data was taken before athletes and non-athletes carried out activities like running and resting. The sampling method was total sampling so that the number of samples to be studied was 20 people with seven professional runner athletes, three junior athletic runners, and ten nonathletes.

Table 4. Participants

\begin{tabular}{|c|c|c|c|c|c|c|c|c|c|c|}
\hline \multirow[b]{2}{*}{ Var } & \multicolumn{7}{|c|}{ AGE } & \multirow{2}{*}{$\begin{array}{l}\mathrm{R} \\
\mathrm{T}\end{array}$} & \multicolumn{2}{|c|}{ t } \\
\hline & $\begin{array}{l}1 \\
6\end{array}$ & $\begin{array}{l}1 \\
7\end{array}$ & $\begin{array}{l}1 \\
8\end{array}$ & $\begin{array}{l}1 \\
9\end{array}$ & $\begin{array}{l}2 \\
0\end{array}$ & $\begin{array}{l}2 \\
1\end{array}$ & $\begin{array}{l}2 \\
2\end{array}$ & & $\begin{array}{l}\mathrm{s} \\
\mathrm{m} \\
\mathrm{a}\end{array}$ & $\mathrm{pt}$ \\
\hline $\begin{array}{c}\text { Profesi } \\
\text { onal } \\
\text { Athlete } \\
\mathrm{n}=10\end{array}$ & 0 & 2 & 1 & 0 & 0 & 0 & 7 & $\begin{array}{c}20 \\
.0 \\
6\end{array}$ & 3 & 7 \\
\hline $\begin{array}{c}\text { Non } \\
\text { Athlete }\end{array}$ & 0 & 0 & 0 & 0 & 0 & 3 & 7 & $\begin{array}{l}21 \\
.0 \\
7\end{array}$ & 0 & $\begin{array}{l}1 \\
0\end{array}$ \\
\hline
\end{tabular}


The data taken includes three things, namely the heart rate before running (rest), during running, and after running or recovery time. Heart rate before running is done in the morning before the athletes warm-up and do activities, and the heart rate data was recorded by the sensor for 5 minutes. Furthermore, data collection was carried out when the athlete ran in the field, and when the athletes finished running. The athletes' data collection was conducted in each participant's data before the exercise, during the exercise, and the break time was counted in 1 session for each athlete, after that, the next athlete's turn.

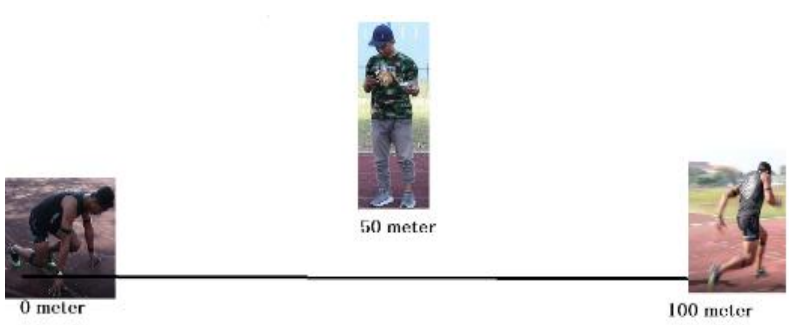

Fig 16. scenario get the data

Figure 16 shows scenarios performed for retrieving heart rate data and motion capture data are carried out in the field with athletes who have paired a heart rate sensor and motion capture at the start and finish position with a distance of $100 \mathrm{~m}$, and the receiver sensor is placed in the middle of the track. Athletes and non-athletes will run for $100 \mathrm{~m}$ distance. The athletes' movement and heart rate data will be recorded in real-time in the field.

\section{Heart Rate Before Exercise.}

After taking the Heart Rate data on 10 the athletes, the data obtained is in the graph below. In figure 17, it can be seen that the average distribution of heartrate at work before doing sports activities is on a scale of 40-66 BPM. Next, the data retrieval with the same procedure on the nonathletes with the number of samples 10 and it is found the results as in Figure 17.

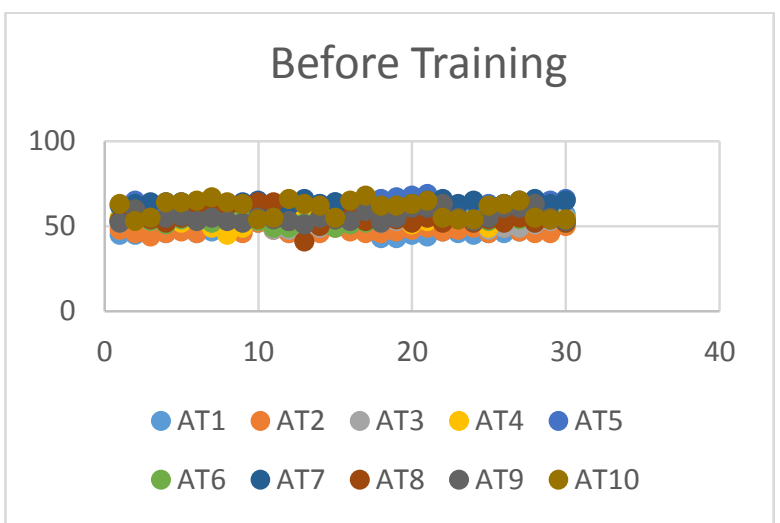

Fig 17. Heart rate athletes before exercise

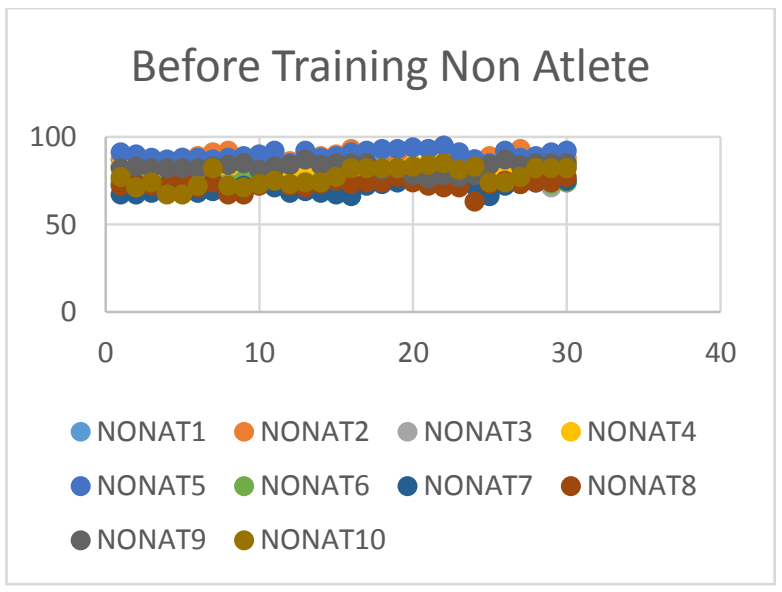

Fig 18. Heart rate nonathletes before exercise

In figure 18 , it can be seen that the distribution of non-complete heart rates in the range of 60-98 BPM is much higher than an athlete with 40-66 BPM. If the results between non-athletes and athlete are combined it will be like in figure 19 .

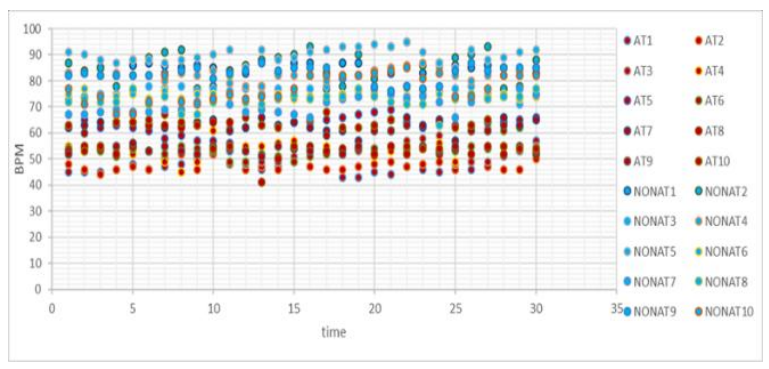

Fig 19. Heart rate athlete and non-athletes before exercise 
In figure 19, its known that the athletes' Heartrate tends to be lower than the nonathletes'. The intensity of exercise affects the space in the heart so that more volume of blood is pumped throughout the body[4][22]. The average of athletes and non-athletes heart rate is $55,28 \mathrm{bpm}$ for athletes and $79,63 \mathrm{bpm}$ for non-athletes.

\section{Heartrate When Running 100m.}

At the time of exercise, an athlete experiences an increase in heart rate due to physical activity.

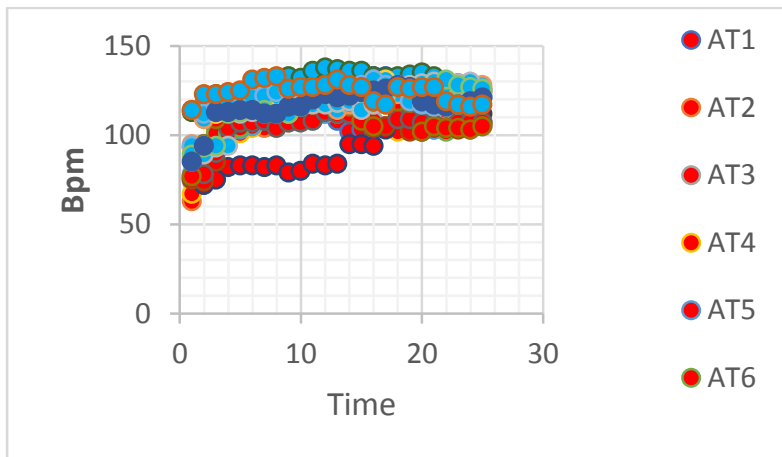

Fig 20. Heart Rate athlete

In Figure 20. We can see that athletes experience a significant increase in heart rate from $60 \mathrm{bpm}$ to $120 \mathrm{bpm}$. This increase in heart rate is triggered by a physical activity carried out by the participants. This heart rate describes the surge in athletes' heart rate when running relatively stable for every second. It shows the distribution of athlete and nonathlete heart rates when running. For nonathlete participants, heart rate tends to be higher than athletes, the initial number while they are running is $80 \mathrm{Bpm}$ and can go up to $140 \mathrm{Bpm}$.

\section{Heartrate After running / resting.}

Heartrate resting is the number of heartbeats at rest after exercise. In figure 21, data from the recording of the sensor heart rate and the distribution of heart rate athletes is known when they have finished exercising.

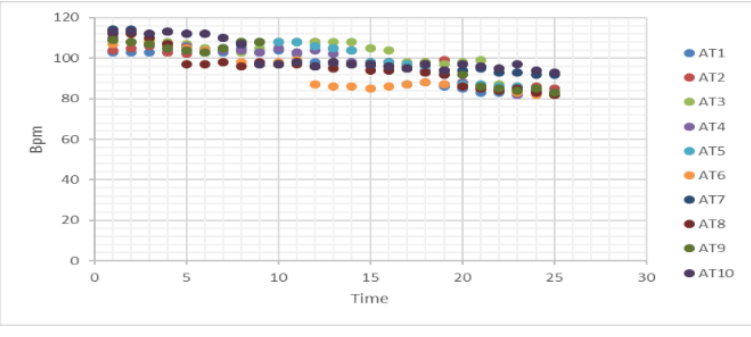

Fig 21. athlete's heart rate when resting

Figure 22 shows the data of heartrate athletes and non-athletes when they have finished exercising, from the data it is known that the level of athletes' heart rate is lower compared to non-athletes. This is caused by intensely exercising in cardiographic athletic skills.

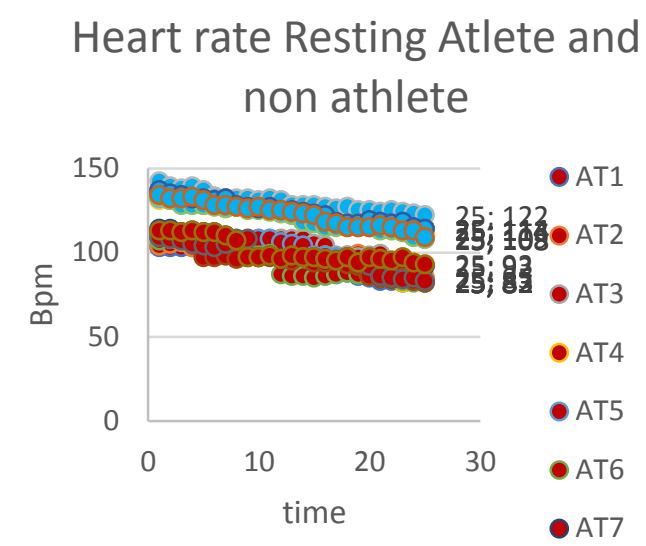

Fig 22, Athletes and non-athletes heart rate when resting

\section{Comparison between athlete and non-athlete Heartrate}

Based on the spread of athlete and nonathlete heart rate data before exercise, during the exercise and rest time. Thus, it can be concluded that an athlete has a heart rate that is more intense than non-athlete in the range of 40-60 Bpm when they take a break. While during the running time, an athlete can increase his heart rate significantly but tends to be stable. is useful to get speed and power at the start of running, because running for a distance of 100 meters requires speed and strength at the start. 


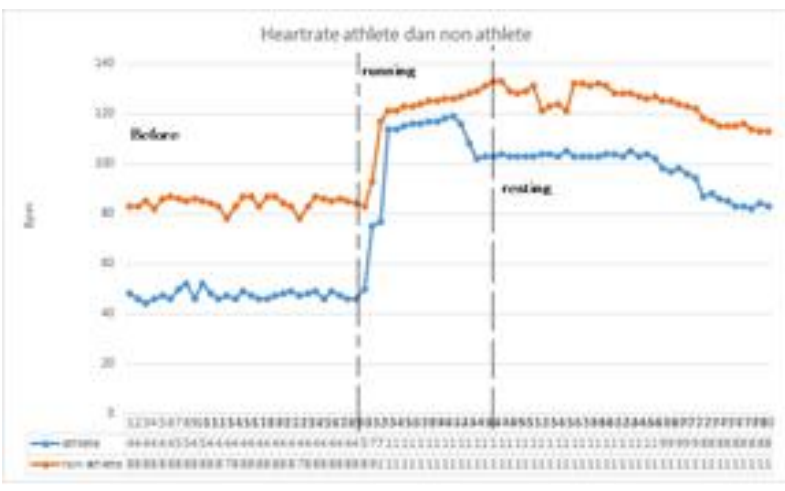

Fig 23. Comparison between athletes and nonathletes heart rate.

Based on Figure 23, it can be concluded that athlete heart rate is lower and stable than non-athletes, this is influenced by intensive exercise.

\section{Results of recording the Wearnotch sensor data.}

Before recording an athlete's data, the athlete must be in a straight position, so that the motion capture sensor can be calibrated with the athlete's shape and posture. After performing the calibration, as shown in figure 24 , the athlete will take the position to prepare to run. The process of recording the athlete's movements starts before and after running.

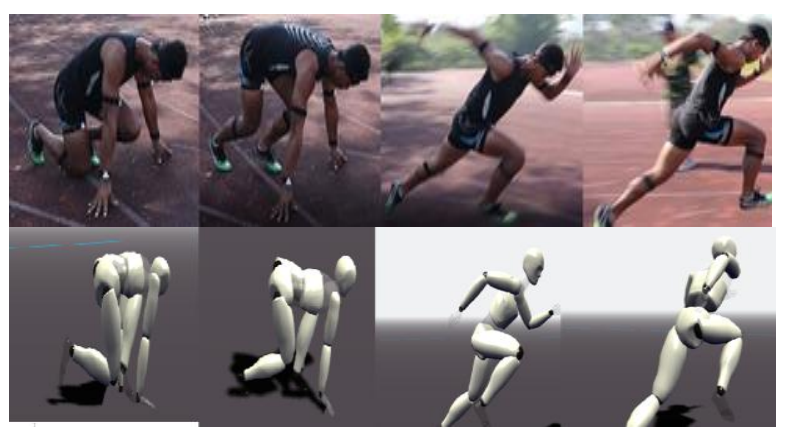

Fig 24. Athletes start and running position.

From the trials that have been conducted, we obtained 2 data, namely sensor data and motion angle data. In the sensor data, there is a gyro and accelerometer information on the $\mathrm{x}, \mathrm{y}$ and $\mathrm{z}$ axes. Whereas in the angular data there is different information on each body part. According to the module from Sports Mechanics, the success of running is determined by the ability of the runner to combine the two limbs, both arms, trunk etc into a unified coordinated motion. Based on the module, the data analyzed is only in some parts: Chest, Knee, Elbow, Hip, Arm, Head

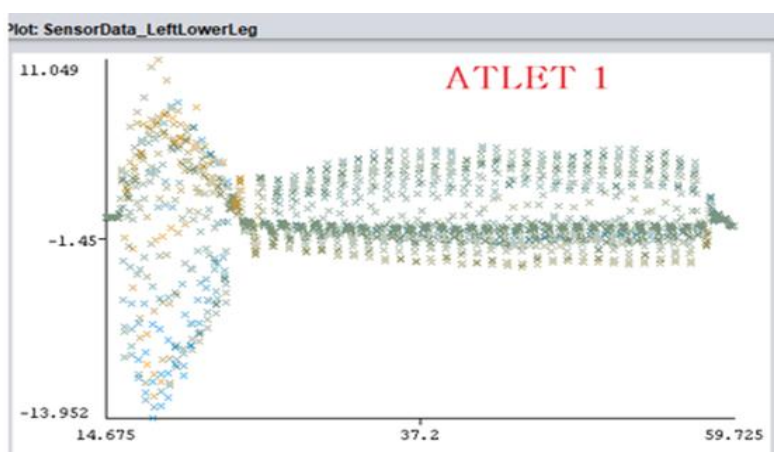

Fig 25. Motion graph data

Wearnotch data that goes into the scrapping database so that it is entered into the Smart Athletics platform database.

The wearnotch sensor sends an average of 98 data in 3 seconds. In other words, in every second the wearnotch can send 32.6666 data. So if we do a record for 1 minute, then the data will be recorded in the amount of +/- 1920 data.

\section{Athlete performance monitoring website}

The web interface serves as the main control for user management on the Platform[23]. In this web interface, the admin can add athletes and trainers, then associate the trainer with athletes. To display the web interface is made as simple as possible to facilitate use by the admin or trainer. There are several pages in this web application, including dashboard pages, user data management, and displaying motion data taken from cloud learning services. The following is the appearance of the web interface that has been created.

The start page when the first user accesses the website page. The start page of the website contains information about athlete training, nutrition, schedules and many more.

On the dashboard page, a summary of the amount of data registered in the database is displayed. On this page, there is also a feature to do Fetch / Synchronization data from the Wearnotch cloud server. When the Sync data button is clicked, the web will update the value 
on the "DO_FETCH" redis key to 1, which will tell the application trigger to carry out its task.

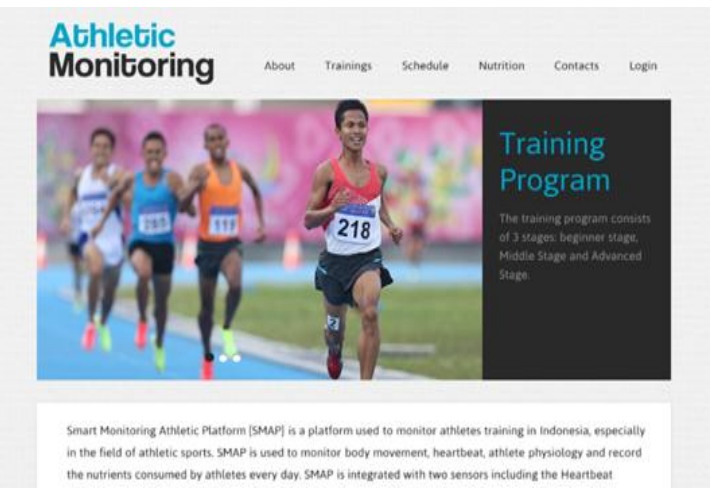

Fig 26. Dashboard Smart Athletic Platform

In addition, there is a menu for setting the current athlete, which will store the user_id user in the Redis shared data. After selecting the athlete's name on the list, then click Submit, the web application will update the data on Redis.

The history page displays motion data obtained from the Wearnotch cloud service. This page shows the name, gender and age of the athlete. To see the motion, you can click the link in the motion data column, as well as download the raw data through the link in the Detail column. To obtain age data, the date of birth is calculated by using Carbon library at Laravel. The function is made into an accessor at laravel ORM, eloquent. With this accessor, a model can have an additional method as specified by the developer.

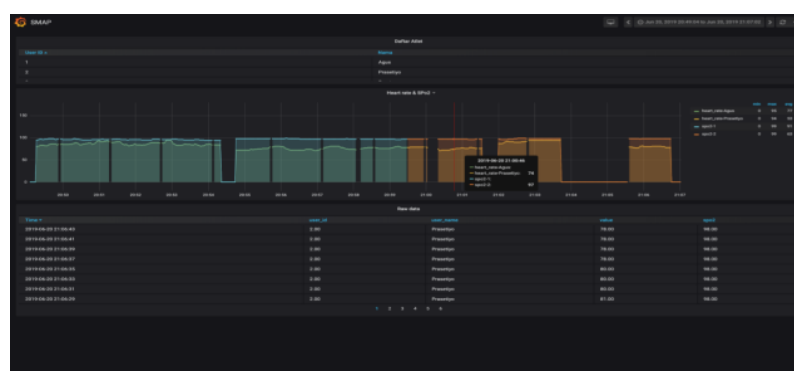

Fig 27. Real-time data sensors.

The dashboard is used to visualize data in real-time from data originating from the sensor heart rate. After the data enters the broker's MQTT and is stored on influx DB, grafana will periodically query influx DB according to the refresh rate setting on the dashboard.

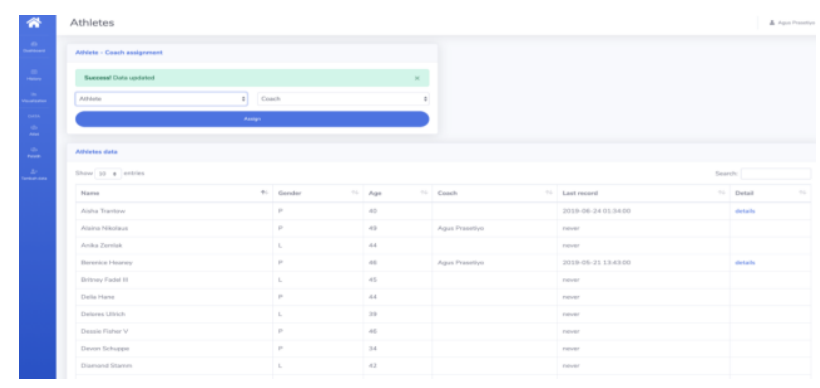

Fig 28. Athletes list page

On this page, the admin can do assignments or delegations between athletes and coaches. Admin only needs to choose the name of the athlete and the coach then clicks the "assign" button. The assign queries whether the database assignment has a coach for the athlete, if it has, it will update the existing data, if not, it will create a new entry.

\section{CONCLUSION}

The athletes' performance monitoring platform using heart rate sensors and motion capture can work well.

The concept of utilising multimode clustering on a server makes possible to record data without worrying about memory capacity. The error rate for reading heartrate by using the T34 polar sensor is $0.4 \%$; in other words, this sensor is good for monitoring the heart rate of athletes in the field. The T34 polar sensor can only transfer data up to 70 meters more so that it can only be used in 100 meters running by placing the sensor in the middle of the running track. The Wearnotch sensor can capture the athlete movements well and be stored on the Wearnotch server. Then, we do fetching to pull the Wearnotch data into the athlete's performance monitoring platform. The fetcher application is very helpful in obtaining data from wearnotch sensor recording and linking it to existing athlete data. The connection speed will greatly affect the communication delay between sensors to the server.

This system still needs further development such as the addition of new parameters, not just motion and heart rate parameters, one of which is the addition of parameters and sensors for nutrition, spo2, and psychology. To carry out data retrieval of more than 100 meters, an additional communication device is 
needed or modify the board and microcontroller so that it can be tied to the

\section{REFERENCES}

[1] R. I. Potapenko, Projection of Indonesian Population, vol. 90, no. 6. 2013.

[2] Y. Bahagia, "Athletic Learning," Ministry of Education., pp. 2-94, 2011.

[3] J. J. Hernández Gómez, V. Marquina, and R. W. Gómez, "On the performance of Usain Bolt in the $100 \mathrm{~m}$ sprint," Eur. J. Phys., vol. 34, no. 5, pp. 1227-1233, 2013.

[4] M. Rafi, T. Nurhayati, and D. M. Sari, "Heart Rate Profile of Professional and Amateur Football Athletes in Bandung," J. Med. Heal., vol. 2, no. 2, pp. 798-805, 2018.

[5] A. E. Aubert, B. Seps, and F. Beckers, Heart Rate Variability in Athletes, vol. 33, no. 12. 2003.

[6] I. Puspasari and P. Susanto, "Monitoring Target Heart Rate (THR) for Optimization," vol. 2, pp. 87-94, 2018.

[7] M. Li and Y. T. Kim, "Design of a wireless sensor system with the algorithms of heart rate and agility index for athlete evaluation," Sensors (Switzerland), vol. 17, no. 10, 2017.

[8] M. H. Berlian et al., "Design and implementation of smart environment monitoring and analytics in real-time system framework based on internet of underwater things and big data," Proc. - 2016 Int. Electron. Symp. IES 2016, pp. 403-408, 2017.

[9] Arridha, R., Sukaridhoto, S., Pramadihanto, D., \& Funabiki, N. Classification extension based on IoTbig data analytic for smart environment monitoring and analytic in real-time system. International Journal of SpaceBased and Situated Computing, vol 7, no 2, pp. 82-93, 2017.

[10] A. Basuki, M. Zikky, J. Akhmad, N. Hasim, and N. I. Ramadhan, "Motion Sensor With Leap Motion To Help Deaf," vol. 8, no. 1994, pp. 317-321, 2016. athlete's body and can be carried when they are running.

[11] ITU

Telecommunication

Standardization Sector (ITU-T), "Recommendation ITU-T Y.2060: Overview of the Internet of things," Ser. Y Glob. Inf. infrastructure, internet Protoc. Asp. next-generation networks - Fram. Funct. Archit. Model., p. 22, 2012.

[12] D. G. V. R. R. C. H. V. V. N. S. Kumar, "Hadoop Distributed File System and Map Reduce Processing on Multi-Node Cluster," Int. J. Sci. Res., vol. 4, no. 8, pp. 1424-1430, 2015.

[13] "Pengembangan Virtual Appliance Server Dengan Metode Virtualisasi," JNTETI (Jurnal Nas. Tek. Elektro dan Teknol. Informasi), vol. 1, no. 1, 2012.

[14] "Comparative Analysis Of Proxmox VE And Xenserver As Type 1 Open Source Based Hypervisors," Int. J. Sci. Technol. Res., vol. 7, no. 3, pp. 72-77, 2018.

[15] D. Soni and A. Makwana, "A survey on mqtt: a protocol of internet of things(IoT)," Int. Conf. Telecommun. Power Anal. Comput. Tech. (Ictpact 2017), no. April, pp. 0-5, 2017.

[16] A. M. author Brownridge, "Real-time motion capture for analysis and presentation within virtual environments," no. June, 2014.

[17] M. U. H. Al Rasyid, F. A. Saputra, and A. Fahmi, "Performance Evaluation of Beacon-Enabled Mode for IEEE 802.15.4 Wireless Sensor Network," Emit. Int. J. Eng. Technol., vol. 1, no. $1,2017$.

[18] R. Rai and P. Chettri, "NoSQL Hands On," in Advances in Computers, 2018.

[19] "Formal Analysis Of Use Case Diagrams," Comput. Sci., vol. 11, no. 1, pp. 115-115, 2010.

[20] H. Andrian, B. Irawan, and A. B. Osmond, "Aplikasi Penghitung Denyut Jantung Berbasis Android," $e$ Proceedin, vol. 2, no. 2, pp. 34863493, 2015. 
[21] M. Rahul, "Review on Motion Capture Technology," Glob. J. Comput. Sci. Technol. F Graph. Vis., vol. 18, no. 1, pp. 1-5, 2018.

[22] F. S. Martinelli et al., "Heart rate variability in athletes and nonathletes at rest and during head-up tilt," Brazilian
J. Med. Biol. Res., vol. 38, no. 4, pp. 639-647, 2005.

[23] A. V. Hausman and J. S. Siekpe, "The effect of web interface features on consumer online purchase intentions, " $J$. Bus. Res., vol. 62, no. 1, pp. 5-13, 2009. 